\title{
Completion of designing and manufacturing of the coil support structure of W7-X
}

\author{
Didier Chauvin ${ }^{\mathrm{a}}$, Torsten Koppe ${ }^{\mathrm{a}}$, Antonio Cardella ${ }^{\mathrm{a}}$, Bernd Missal ${ }^{\mathrm{a}}$, Dirk Pilopp ${ }^{\mathrm{a}}$, Giovanni Di \\ Bartolo $^{b}$, Rocio Camín ${ }^{c}$, Ivan Gonzales ${ }^{c}$, Luca Giordano ${ }^{d}$, Stefano Langone, \\ ${ }^{a}$ Max-Planck-Institut für Plasmaphysik, Teilinstitut Greifswald, Wendelsteinstr. 1, D-17491 Greifswald, Germany \\ ${ }^{b}$ M\&G srl Consultants, Via Dei Romanelli 8, I-20034 Giussano, Italy \\ ${ }^{c}$ Equipos Nucleares S.A., Avda. Juan Carlos 1, 39600 Maliano, Spain \\ ${ }^{d}$ ROVERA Costruzioni Meccaniche, Via Vecchia di Cuneo 45, 12011 Borgo san Dalmazzo, Italy \\ ${ }^{e}$ Romabau Gerinox AG, Fohlenweide, CH-8570 Weinfelden/Thurgau, Switzerland
}

In February 2000, the project called coil support structure for the Wendelstein 7-X fusion machine was started. Since October 2009 the full production of this big (80 tons) and complex component is now completed and delivered at IPP Greifswald. The W7-X coil system consists of 20 planar and 50 non-planar coils. They are supported by a pentagonal $10 \mathrm{~m}$ diameter, $2.5 \mathrm{~m}$ high called coil support structure (CSS). The CSS is divided into five modules and each module consists of two equal half modules around the radial axis. Currently, the five modules were successfully assembled with the coils meeting the tight manufacturing tolerances. Designing, structural calculation, raw material procurement, welding \& soldering technologies, milling, drilling, accurate machining, helium cooling pipe forming, laser metrology, ultra sonic cleaning and vacuum test are some of the key points used all along this successful manufacturing process. The lessons learned in the large scale production of this difficult kind of support structure will be presented as relevant experience for the realization of similar systems for future fusion devices, such as ITER.

Keywords: Designing, Mechanical analysis, Manufacturing process, Accurate machining.

\section{Introduction}

Wendelstein 7-X (W7-X) is a fully optimized lowshear stellarator and shall demonstrate the reactor potential of this fusion plant. It is presently under construction at the Greifswald Branch Institute of MaxPlanck-Institut für Plasmaphysik (IPP). The superconducting magnet system will allow continuous operation, limited only by the plasma exhaust system whose capacity is designed for $30 \mathrm{~min}$ full power operation. The Wendelstein 7-X (W7-X) coils and structures are part of the largest superconducting fusion device being constructed at present. They have represented a technical challenge at industrial level and required proven techniques and manufacturing processes in accordance to the highest quality standards. The production of component such as CSS has required a management of monitoring for quality and tests.

\section{Designing and mechanical analysis}

The main supporting structure of the superconducting magnets of the Wendelstein 7-X Stellarator under construction in Greifswald consists of a stainless steel, AISI 316 LN ring structure, which is divided in five modules. Each module is subdivided in two half modules (HMs) for assembly reasons as shown in Fig. 1. The ring structure of CSS and its connections were designed before the start of the construction on the base of the results of a complex but partial structural analysis model, using anti-symmetric boundary conditions in order to limit the model size. Two robust vertical/radial flanges (see also in Fig. 2) bolted together with M45 studs will be used for the connection between modules. The connection between the HMs, which has to resist higher loads, was designed using steel step flanges, as shown in
Fig. 2, mating with the corresponding step flange of the adjacent HM, and using M45 studs.

More accurate analyses using global models of the magnetic system found out (2004-2006) that the loads on the connections were much higher than previously computed. The load increase was mostly compensated by using high strength Inconel ${ }^{\circledR} 718$ studs and tensioners. However, a component of the loads tends to warp the ring structure at the half module flange connection and for the most severe load conditions the flanges slightly slip and start to open. In order to solve the problem without radically changing the design of the CSS in advanced status of manufacturing, a solution has been proposed, which makes use of a selected number of studs as shear dowels. In addition to the friction forces of the standard bolted connection, the shear dowels resist the shear loads and limit the warping. The use of shear dowels requires a precise mating with the flange holes. This has required resolution of several operations such as reaming together flange holes. The development of the CSS has continued using full and reduced scale experiments and FE models. Design changes have already been implemented based on the outcome of the experiments and models. The CSS manufacturing has reached a phase where many support elements have been installed for the first time. This has required closure of several technical issues that the program faced in the recent last years. The numerous design and test activities on the various support elements have provided a solid basis for ensuring that the CSS will perform reliably throughout the experimental life of the W7-X stellarator [1]. 
The objective of the CSS calculation project was to create a finite element model of one half-module and analyze its mechanical behaviour under loads resulting from three plasma scenarios with preliminary bolt tightening and cooling down from 295 to $4 \mathrm{~K}$. Finite element computations were carried out in order to obtain the complex stress distributions inside the CSS. The maximum stress and strain values can be kept within technical limits by the current design of CSS.

\section{Organisation of the manufacturing}

The CSS detail design has been subjected to considerable modifications over the years, since 1999. Thanks to a strong collaboration between IPP and several sub-contractors, the design of CSS has been continuously improving till it fulfils the requirement of dimensional accuracy, and also complies with the optimized capacity of production for casting, machining, welding and cleaning activities. A group of industrial company such as ENSA (Spain), ROVERA (Italy), ROMABAU-GERINOX (Switzerland), M\&G.srl (Italy), has participated to the manufacturing of all 10 half module of CSS. Logistical tasks are one of the difficulties of the project, because during all manufacturing steps each half-module has to cover almost $7000 \mathrm{~km}$ before to be delivered at IPP Greifswald.

In order to compensate intrinsic difficulties due the remoteness between different subcontractors, a clear documentation, the quality of drawings, work culture, a permanent communication and management are really fundamental to obtain a good quality of manufacturing which complies with delivery schedule. For improving the follow-up and advancement of manufacturing tasks, the CSS production has been supervised by IPP but also supported by external inspectors. Indeed, the role of M\&G's inspectors was essential for following-up the contract in compliance with the highest standards of quality assurance. Purpose of these inspections is also to validate respective rules, norms and regulations inherent in the step by step manufacturing of CSS. The experimental life of CSS manufacturing concerns also the cleaning. Since June 2008, the company RomabauGerinox has been engaged to enhance the cleanliness of each HM by means of ultrasonic cleaning. This additional tasks has modified the organisation and of course the logistic of the project. The last organisation is described in Figure 3.

\section{Technical specifications for manufacturing of CSS}

\subsection{Standards in quality assurance}

IPP technical specifications inherent in the step by step manufacturing of CSS should complies with rules, norms and regulations. For instance, general tolerances are defined for linear and angular measurements on norm DIN ISO 2768-m, see also picture in figure 4.
According to DIN ISO 2768-m general tolerances for linear measures and level squares with four tolerance classes are useful for simplifying drawings. The tolerance class precision levels common in workshops should be used. If smaller tolerances are needed or bigger ones are more economical, then these tolerances are indicated next to the nominal size.

\subsection{Materials of CSS}

Properties and stress/strain curves for each material are listed in the following sections. The material properties are defined at 295, 77 and $4 \mathrm{~K}$, and are linearly interpolated in between. The part material attribution is presented in Fig.5. The plates of the ring including reinforcement plates, the extensions and the shims are made of DIN 1.4429 stainless steel.

On receiving all materials, semi finished products and prefabricated parts, the company ENSA and his subcontractors ROVERA had to issue certificates. All material and batch numbers and all needed properties must be included in these certificates. In addition, IPP is continuously performing an independent analysis of the amount of cobalt in the stainless steel which is limited to a maximum of $500 \mathrm{ppm}$.

\subsection{Welding works of HMs frame and extensions}

Generally, the quality of a weld joint is directly influenced by the welding input parameters during the welding process; therefore, welding can be considered as a multi-input multi-output process. Unfortunately, a common problem that we had to face with our manufacturer ENSA was the control of the process input parameters to obtain a good welded joint to bead geometry and weld quality while having minimal detrimental residual stresses and distortion. It has been necessary to determine the weld input parameters for every new welded product to obtain a welded joint with the required specifications. To do so, it requires a timeconsuming trial and error development effort, with weld input parameters chosen by the skill of the engineer or machine operator. DIN EN 288 specifies how a welding procedure specification is qualified by welding procedure tests, ranges of qualification and acceptance levels. In that way, we have developed all necessary welding qualified procedures according to requirements based on the related DIN/EN rules. These DIN rules describe the good practice work which has be performed from the companies. In IPP supply contract it is requested to ENSA to work according to the scientific and technologic state of art. All along the manufacturing of each HM of CSS, several tests were performed: visual check, test of welding seems, measurement after heat treatment, permeability, ultra sonic controls, dye penetrant test, X-rays, as shown in Figs 6 and 7.

$$
) \text {. }
$$

The purpose of these tests in the detection of crack, surface pore, lack of fusion, incomplete root penetration, undercut (continuous and intermittent), shrinkage groove, excess weld material, excessive convexity, 
excess penetration, incorrect weld toe, overlap, burn through, asymmetry of fillet weld, root concavity or porosity, poor restart, insufficient throat, which are among the possible non-conformances detected during test.

\subsection{Accurate machining}

Accurate machining was performed by Rovera (Italy). Since 2005, an important background historical was developed in order to improve the final machining of CSS. Multi-axis NC machines have vast applications in modern industry especially in machining work pieces with sculptured surfaces such as CSS. We had to face with more choices of machining parameters such as the setup of the work piece on the mounting table, and positioning of the cutter. Different combinations of machining parameters might produce large variations in the final product quality. At Rovera the CAM software has proposed a systematic method of determining optimal machining parameters. Experimental studies before actual machining are time consuming and not cost-effective. Machining accuracy is related to a set of pre-determined parameters such as the parameters of the cutter, positioning of the cutter, and cutter orientation. For the CSS we focused on geometric machining parameter optimization to improve the machining accuracy. The problem of geometric machining parameter optimization is formulated as a constrained nonlinear programming problem. For the CSS the goal was to maximize the similarity between the desired surface and the actual surface under constraints such as non-interference conditions, geometrical design requirements of the cutter, and scallop height requirement determined by IPP. For technical and financial reasons of production, a strong and permanent technical collaboration between IPP, ENSA and ROVERA was necessary in order to:

- Propose and agree changes of the HM design related to technical difficulties and extra cost processes.

- For each of the processes involved, propose a process of estimates and principles for the associated tooling.

- Validate the choice of coupled material/machining process with regard to the geometry and specifications for producing each HM.

- Specify the equipment required for the production: machine-tool (gantry machine at Rovera), tool and tooling.

- Establish documents, procedures for machining and quality control (technical documentation).

The numerous design and test activities have provided a solid basis for the feedback of big pieces like the 10 half modules composing the coil support structure. All along these technical experiences, the machining process had been then developed, and successfully carried out.
Accurate machining was validated through dimensional measurements in sensitive areas for holes of the three steps flanges, extensions or module flanges as shown in Fig.7.

Fig 7. Accurate machining at Rovera (Italy).

\subsection{Cooling pipe assembly}

Bending, cutting, milling, welding, soldering and testing tasks were necessary for a complete cooling pipes assembly. Helium cooling pipes used during the manufacturing are seamless and from DIN 1.4571 materials. For each half module, $45 \mathrm{~m}$ of copper strip (20 $x$ 1) and $49 \mathrm{~m}$ of DIN 1.4571 pipes were needed. This task was performed by ENSA as shown in Fig 8.

Fig 8. Cooling pipe assembly performed at ENSA (Spain).

The duration of cooling pipes activities needed 4 weeks, including also helium pressure test (30 bars) and the helium detection. An orbital welding device is also used for connection of each pipe, but so far only visual checks were performed.

\section{Results and conclusion.}

Because of a strong collaboration between IPP and several sub-contractors, the design and manufacturing is now completed. The CSS design has been continuously improved till it fulfils the requirement of dimensional accuracy. It also complies with the optimized capacity of production, machining, and assembling activities. In order to compensate intrinsic difficulties due to the remoteness between different subcontractors, a clear documentation, the quality of drawings, the work culture, a permanent communication and management are really fundamental to obtain not only a good quality of manufacturing but an acceptable manufacturing process. For improving the follow-up and advancement of manufacturing tasks, the CSS production was supervised by IPP but also supported by external inspectors. The feedback in designing and manufacturing of CSS is a typical example of difficulties expected in ITER. The work of the responsible officer, in particularly in the field of big components manufacturing such as W7-X coil support structure requires, of course, technical and engineering skills but not only: versatility, good analytical capabilities, communication skills, organisational skills and ability to work under pressure in a multicultural international setting are prevailing.

\section{References}

[1] J. Reich, Manufacture of Cryostat components for Wendelstein 7-X, Fusion Eng. Des. 69 (2003) 345-3 